\title{
FUNCIONALIZACIÓN DE PRÓTESIS POLIMÉRICAS POR PROYECCIÓN TÉRMICA: UNA REVISIÓN
}

\author{
Benjamín Ortega ${ }^{1 *}$, Hannia Gonzalez ${ }^{2}$
}

1: CIATEQ A.C. Av. Manantiales 23-A, Parque Industrial Bernardo Quintana, El Marqués, Querétaro, México

2: Universidad Tecnológica de Corregidora, Facultad de Biotecnología, Querétaro, México.

* E-Mail: benjamin.ortega@ ciateq.edu.mx

\begin{tabular}{|c|c|}
\hline PALABRAS CLAVE & RESUMEN \\
\hline $\begin{array}{l}\text { Bioactividad } \\
\text { Prótesis poliméricas } \\
\text { Implantes } \\
\text { Proyección térmica }\end{array}$ & $\begin{array}{l}\text { Se presenta una revisión de la literatura acerca de la funcionalización } \\
\text { de materiales poliméricos para su uso en aplicaciones ortopédicas por } \\
\text { proyección térmica. Los recubrimientos bioactivos sobre materiales con } \\
\text { base polimérica son una alternativa desarrollada con el propósito de } \\
\text { mejorar las propiedades superficiales de estos materiales. La } \\
\text { modificación superficial mediante la incorporación de un material } \\
\text { bioactivo es una opción atractiva para mejorar el desempeño de } \\
\text { materiales al ser implantados en el organismo. Primero se describen de } \\
\text { manera breve los conceptos referentes a la proyección térmica. } \\
\text { Después, se exploran los principales polímeros empleados en el ámbito } \\
\text { de prótesis y los biomateriales empleados en la fabricación de los } \\
\text { recubrimientos bioactivos. Además, se describen los principales } \\
\text { parámetros que afectan las propiedades del polímero. Esta revisión } \\
\text { tiene como objetivo resumir las contribuciones más recientes e } \\
\text { importantes en el tema de funcionalización de prótesis poliméricas } \\
\text { modificadas superficialmente por proyección térmica. }\end{array}$ \\
\hline
\end{tabular}

FUNCTIONALIZATION OF POLYMERIC PROSTHESES BY THERMAL SPRAY: A REVIEW

\begin{tabular}{ll}
\hline KEYWORDS & ABSTRACT \\
\hline Bioactivity & A review on the functionalization of polymeric materials employed \\
Polymeric prostheses & in orthopedic applications by thermal spray is presented. The \\
Implants & bioactive coatings on polymeric-based materials have been developed \\
Thermal spray. & aiming to improve their surface properties. Surface modification \\
& through the incorporation of a bioactive material is an attractive \\
option to improve the performance of materials when implanted in \\
the body. In this review, first, the concepts related to thermal spray \\
are briefly described. Then the main polymers used in prosthetics \\
field and the biomaterials used in the manufacture of bioactive \\
coatings are explored. In addition, the main parameters that affect the \\
properties of the polymer are described. This review aims to \\
summarize the most recent and important contributions to the \\
functionalization of polymeric prostheses superficially modified by \\
thermal spray techniques.
\end{tabular}




\section{INTRODUCCIÓN}

En las últimas décadas se han desarrollado biomateriales para ser empleados en aplicaciones ortopédicas de carga, dentro de los cuales, los metales se han establecido como los materiales más empleados en este campo, sin embargo, estos presentan algunas desventajas como lo son las partículas generadas por desgaste, la fuga de iones, apantallamiento de esfuerzos (debido a la diferencia entre el módulo elástico de los metales y el hueso), y algunos problemas asociados al diagnóstico clínico. Esto ha generado la necesidad de desarrollar materiales que tengan cada vez mejor desempeño dentro del organismo, lo cual, ha propiciado que el uso de materiales poliméricos en aplicaciones de ortopedia haya aumentado en los últimos años. Sin embargo, los polímeros, al igual que los metales empleados en este tipo de aplicaciones, son bioinertes al entrar en contacto con el medio biológico, por lo que, es necesario modificar su superficie para dotar a estos materiales con propiedades bioactivas.

Dentro de las técnicas de modificación de superficie, la proyección térmica se ha posicionado ampliamente como una opción promisoria para la fabricación de recubrimientos que provean a los materiales poliméricos con propiedades bioactivas en su superficie [1-5]. La proyección térmica comprende un conjunto de procesos en los que, mediante la implementación de energía química, eléctrica o cinética, el material seleccionado para formar el recubrimiento es generalmente procesado en forma de polvo. En estos procesos, las partículas de material seleccionado son aceleradas a través de una boquilla y son proyectadas hacia un sustrato para formar eventualmente un recubrimiento.
Existen varias técnicas dentro de la proyección térmica, distinguiéndose una de otra principalmente por el tipo de energía utilizada para tratar termo-cinéticamente las partículas del material seleccionado para formar el recubrimiento (existen otras diferencias en los procesos, como el medio en que se realiza, la forma en que se encuentra el material a proyectar, entre otros). Por ejemplo, se conocen las técnicas de proyección térmica por combustión en donde la fuente de energía de estos procesos se obtiene a partir de una reacción de combustión resultante entre un combustible (i.e. por lo general hidrocarburos $\mathrm{C}_{\mathrm{x}} \mathrm{H}_{\mathrm{y}}$ o en ocasiones hidrógeno) y oxígeno. La proyección térmica por combustión de baja velocidad (FS, siglas en inglés), la proyección térmica por combustión de alta velocidad (HVOF, siglas en inglés) y la proyección térmica por detonación (D-Gun, siglas en inglés) son las principales técnicas pertenecientes a la proyección por combustión. Dentro de las técnicas que emplean como fuente de energía la descarga eléctrica, se incluyen la proyección térmica por plasma por corriente directa, el plasma acoplado inductivamente por radio frecuencia, la proyección térmica por arco eléctrico y el plasma de arco transferido. En estos procesos la descarga eléctrica se logra empleando arcos con diferentes tipos de antorchas, pero el principio básico se basa en la presencia de un ánodo y un cátodo mediante el cual se forma un arco eléctrico, el cual calienta a un gas formador de plasma a través del cual se proyectan partículas a altas temperaturas. Por último, en la proyección térmica empleando energía cinética, conocida comúnmente como proyección en frío (CS, siglas en inglés) tiene como principio de funcionamiento la inyección de un gas a alta presión el cual es transportado a través de una boquilla convergente-divergente en donde este se comprime y se expande generando un flujo de gas de alta velocidad. El flujo de gas 
a alta velocidad es empleado para la aceleración de las partículas en el proceso de CS. Al igual que en otros procesos de proyección térmica, las partículas del material seleccionado para formar el recubrimiento son tratadas termocinéticamente en el proceso, sin embargo, en CS, la relación entre energía cinética y energía térmica es mucho mayor que en otros procesos de proyección térmica, por lo que, generalmente se considera como un proceso en estado sólido, empleando así comúnmente materiales dúctiles que permitan la formación de un recubrimiento por deformación plástica sucesiva de partículas. Dentro de este conjunto de procesos se encuentran la proyección en frío de alta presión (HPCS, siglas en inglés) y de baja presión (LPCS, siglas en inglés) $[6,7]$.

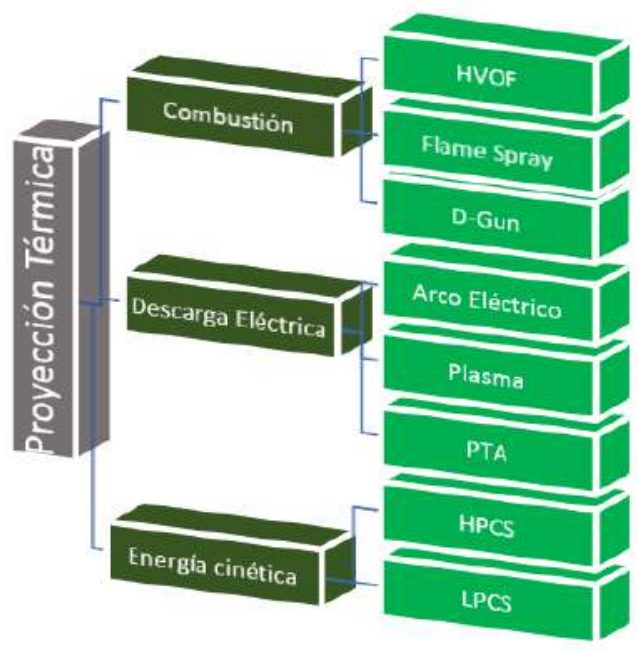

Figura 1. Esquema general de proyección térmica

Los procesos de proyección térmica permiten la selección de distintos tipos de materiales para la formación de un recubrimiento, como metales, cerámicos, polímeros y compuestos, además de que también permite la selección de una amplia gama de materiales como sustrato, incluyendo cerámicos, metales, y polímeros, lo cual, hace que la proyección térmica sea muy atractiva para gran variedad de sectores industriales como lo son: el aeroespacial, biomédico, automotriz, textil, entre otros; sectores en donde lo que se pretende principalmente es mejorar el desempeño funcional, extender la vida o reducir costos de componentes mediante la aplicación de un recubrimiento [6, 8, 9]. En particular, los materiales poliméricos han sido empleados en proyección térmica tanto como material de recubrimiento, así como sustrato. Los materiales poliméricos tienen una amplia gama de aplicaciones, las cuales exceden por mucho las de cualquier otro tipo de materiales, pues esta familia de materiales posee una gran variedad de propiedades, esto es, ser ligeros, flexibles, de fácil manufactura $y$ en general de bajo costo $[8,10,11]$.

En el sector médico, el uso de polímeros para la manufactura de prótesis se debe principalmente a las propiedades mecánicas que presentan algunos de éstos, las cuales son muy similares al hueso, evitando así, el apantallamiento de esfuerzos, fenómeno que puede causar el aflojamiento y eventual fallo de una prótesis [12]. Incluso algunos polímeros presentan módulos elásticos más cercanos al hueso que los principales materiales metálicos empleados hoy en día en prótesis ortopédicas, como lo es el caso del titanio y algunas de sus aleaciones. Además, otra característica atractiva de los materiales poliméricos en el campo médico es su radiolucencia, la cual permite apreciar de mejor manera la interacción del material implantado con el medio biológico mediante Rayos X [13].

En un implante es importante la interacción de su superficie con el medio biológico. En particular, en el caso de los polímeros usados como implantes internos en ortopedia se ha observado que, a pesar de sus propiedades mecánicas y radiolucencia, estos materiales son bioinertes, es decir, después de un tiempo de ser implantados no hay una unión del tejido óseo con el material. Esto promueve la aparición de micro-movimientos causados 
por la actividad física del paciente, lo que, a su vez, promueve la formación de partículas de desgaste debido a la ductilidad característica de este tipo de materiales. Todo este proceso culmina con la aparición del fenómeno de osteólisis en el tejido óseo del paciente. Con este antecedente, la funcionalización superficial de algunos materiales poliméricos, en particular, de aquellos con propiedades mecánicas similares al hueso, resulta ser una alternativa de interés en la industria biomédica [14]. Es importante mencionar que, aunque hay un gran interés por la industria biomédica en mejorar las propiedades superficiales de algunos materiales poliméricos, otro tipo de industrias como la automotriz o la electrónica también consideran la modificación superficial para mejorar algunas propiedades de los polímeros como lo son la conductividad eléctrica y térmica, blindaje electromagnético y proveer protección contra radiación y erosión, entre otros. La mayoría de los recubrimientos aplicados en estas industrias son metálicos y/o intermetálicos, lo que permite el uso de diferentes técnicas distintas a la proyección térmica para su fabricación. Sin embargo, se conoce tradicionalmente en la industria biomédica, que para mejorar la biocompatibilidad y bioactividad de los implantes se emplean comúnmente recubrimientos cerámicos, lo cual resulta ser un reto de fabricación en caso de implantes poliméricos. Por ejemplo, de las técnicas previamente mencionadas (Figura 1), el HVOF y la proyección térmica por plasma atmosférico (APS), han sido procesos que en las últimas décadas se han empleado para la fabricación de recubrimientos biocompatibles y bioactivos sobre implantes metálicos para aplicaciones ortopédicas. Estos procesos se caracterizan por alcanzar temperaturas significativamente más altas en relación con las temperaturas de fusión y degradación de los materiales poliméricos de interés para la industria biomédica. En consecuencia, el reto principal en cuanto al uso del HVOF y del APS para la aplicación de recubrimientos cerámicos bioactivos en polímeros es controlar los parámetros de procesamiento evitando degradar o afectar significativamente las propiedades mecánicas y físicas del implante. Es por ello que, en los últimos años, la técnica CS ha surgido como una alternativa de interés dadas la baja energía térmica involucrada en el proceso en comparación al HVOF y el APS. A pesar de existir algunos trabajos referentes a la fabricación de recubrimientos biocompatibles y bioactivos por proyección térmica sobre sustratos poliméricos aún es un campo que no ha alcanzado completamente su potencial. En el presente trabajo se revisará el estado del arte actual en esta materia, definiendo primero algunos biopolímeros de interés y sus características principales.

\section{BIOPOLIMEROS}

Muchos tipos de polímeros son empleados en dispositivos biomédicos, los que incluyen ortopédicos, dentales, tejido suave, implantes cardiovasculares, etc. Algunos de estos materiales se degradan en el cuerpo (por ejemplo, suturas degradables), mientras que otros pueden mantenerse químicamente estables. Entre los polímeros comúnmente empleados en aplicaciones biomédicas podemos encontrar la goma de silicona (SR, siglas en inglés), polietileno (PE), polipropileno (PP), politetraflouroetileno (PTFE), entre otros. Estos materiales poliméricos se caracterizan por estar formados por largas cadenas de carbono que consisten en un gran número de unidades repetidas. La mayoría de estos biopolímeros presentan un comportamiento termoplástico [15], es decir, cuando este tipo de polímeros son sometidos a calor pueden presentar grandes cambios en sus propiedades mecánicas y físicas. De manera general, es importante conocer el comportamiento térmico de estos materiales con el fin de 
establecer las condiciones de procesamiento ideales, en particular, cuando se usan métodos de modificación superficial como la proyección térmica en donde se ven involucradas altas temperaturas, puesto que se conoce que hay una relación estrecha entre las propiedades mecánicas de los polímeros, el peso molecular y su grado de cristalinidad. $\mathrm{Si}$ las condiciones de procesamiento afectan alguna de estas características, entonces las propiedades mecánicas serán afectadas; esto es muy importante conocerlo puesto que son las propiedades mecánicas de los biopolímeros, por ejemplo, del PEEK o del HDPE muy deseables para aplicaciones ortopédicas, por lo que, durante su procesamiento, en particular, cuando se deposita algún tipo de recubrimiento sobre su superficie, se busca no degradar el material, para no perder las propiedades mecánicas para las que fueron diseñados los implantes.

\section{BIOPOLIMEROS EN PRÓTESIS ORTOPÉDICAS}

El éxito de un biomaterial depende de las propiedades del material, el diseño, y la biocompatibilidad; sin embargo, hay factores que van más allá de la capacidad del ingeniero, pues existen factores fuera de su control como la técnica empleada por el cirujano, la salud y condición del paciente, así como sus actividades cotidianas. Sin embargo, desde el diseño del implante es factible realizar una selección adecuada del material y considerar características anatómicas del paciente para evitar posibles fallos. Se reportan comúnmente fallos en biomateriales como, por ejemplo, una respuesta no deseada, toxicidad, reacciones alérgicas, o incluso la inducción de cáncer. De esta forma, se puede identificar como una propiedad clave la biocompatibilidad. Los materiales biocompatibles no irritan las estructuras circundantes, no provocan reacciones alérgicas, inflamatorias o inmunológicas y no causan cáncer [16]. Otro punto importante, antes mencionado, es que, a diferencia de las prótesis metálicas, algunos de los polímeros empleados en ortopedia no presentan apantallamiento de esfuerzos, por ejemplo, cuando se emplea una prótesis de acero inoxidable (SS) este fenómeno es recurrente [17]. El uso de biopolímeros en ortopedia abarca tanto prótesis externas como implantes. Estos últimos se encuentran en contacto con fluidos corporales y dentro de estos encontramos elementos de fijación, cementos óseos, membranas, componentes de órganos artificiales, implantes craneales, implantes cervicales, copas acetabulares, entre otros.

Los principales polímeros utilizados en aplicaciones ortopédicas en contacto con fluidos corporales son el polietileno de peso molecular ultra alto (UHMWPE), ácido poliláctico (PLLA), polieteretercetona (PEEK), polimetilmetacrilato (PMMA), entre otros $[11,15,18]$. El PMMA es un polímero termoplástico con módulo elástico (1.8-3.1 $\mathrm{GPa})$ cercano al del hueso (10.4-14.8 GPa para hueso trabecular y 18.6-20.7GPa para hueso cortical) $[19,20]$ que es estable hasta $65^{\circ} \mathrm{C}$, sin embargo, mediante la adición de estabilizadores térmicos puede ser estable hasta $100^{\circ} \mathrm{C}$. Éste ha sido empleado en el campo ortopédico como cemento óseo [21]. Por otra parte, para aplicaciones estructurales, las PAEKs son la familia de polímeros más usados en ortopedia, dentro de la cual se encuentra el PEEK. Las poliariletercetonas (PEAKs) son una familia de polímeros termoplásticos aromáticos que consisten en una cadena molecular de anillos con uniones de cetona y éter, esta familia de polímeros es utilizada por sus propiedades fisicoquímicas como rigidez, dureza y también su capacidad de ser esterilizado sin ser degradado. Por ejemplo, uno de los polímeros pertenecientes a las PAEKs es el PEEK, el cual, presenta una estructura molecular que es relativamente rígida debido a la presencia de bencenos en la 
cadena principal. Sin embargo, puede rotar axialmente sobre los enlaces de éter (-O-) o carbono-cetona (-CO-),por lo tanto, la cadena principal no se mantiene estática, sino que vibra y rota, como respuesta a las deformaciones externas que se apliquen o energía térmica por lo que puede adquirir una gran deformabilidad. El PEEK se ha posicionado como el material polimérico más empleado en sustitución de componentes metálicos, esto debido en gran medida, a su módulo elástico $(8.3 \mathrm{GPa})$ cercano al del hueso humano cortical (17.7 GPa), el cual es mucho más bajo que el de otros materiales ampliamente utilizados en aplicaciones como son las aleaciones de titanio (116 GPa) y aleaciones cromo-cobalto (210 GPa)[22, 23]

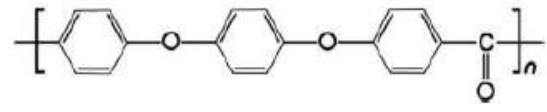

Figura 2. Estructura química del PEEK[14].

El UHMWPE es uno de los materiales más empleados para aplicaciones ortopédicas, su peso molecular y propiedades químicas hacen que este polímero tenga buena resistencia al desgaste y alta dureza. Estas propiedades han hecho que sea utilizado en la fabricación de herramientas para artroplastia. Es empleado en implantes de cadera, en donde ha reemplazado componentes metálicos [24].

Tabla 1. Uso de biomateriales en aplicaciones ortopédicas [15]

\section{IMPLANTES ORTOPÉDICOS}

\begin{tabular}{|c|c|}
\hline Aplicación & Materiales empleados \\
\hline $\begin{array}{c}\text { Dispositivos de unión } \\
\text { ósea y estabilizadores }\end{array}$ & $\begin{array}{c}\text { Acero inoxidable, titanio } \\
\text { y algunas de sus } \\
\text { aleaciones, aleaciones } \\
\text { cromo-cobalto, PEEK, } \\
\text { PLLA }\end{array}$ \\
\hline Articulaciones \\
artificiales & $\begin{array}{c}\text { Acero inoxidable, titanio } \\
\text { y algunas de sus } \\
\text { aleaciones, aleaciones } \\
\text { cromo-cobalto, zirconia, } \\
\text { alúmina, UHMWPE, } \\
\text { silicona }\end{array}$ \\
\hline Ligamentos y & $\begin{array}{c}\text { Acero inoxidable, titanio } \\
\text { y algunas de sus }\end{array}$ \\
\hline
\end{tabular}

\begin{tabular}{|c|c|}
\hline $\begin{array}{c}\text { (suturas no } \\
\text { reabsorbibles, } \\
\text { sistemas de anclaje) }\end{array}$ & $\begin{array}{c}\text { aleaciones, aleaciones } \\
\text { cromo-cobalto, PEEK, } \\
\text { PLLA, PET, UHMWPE, } \\
\text { nylon. }\end{array}$ \\
\hline Dispositivos espinales & $\begin{array}{c}\text { titanio y algunas de sus } \\
\text { aleaciones, aleaciones } \\
\text { cromo-cobalto, } \\
\text { UHMWPE, PEEK. }\end{array}$ \\
\hline $\begin{array}{c}\text { Inductores de } \\
\text { regeneración tisular }\end{array}$ & $\begin{array}{c}\text { Fosfatos de calcio, } \\
\text { autoinjertos, aloinjertos, } \\
\text { colágeno, PMMA. }\end{array}$ \\
\hline
\end{tabular}

\section{MATERIALES EMPLEADOS EN TÉRMICA \\ BIOACTIVOS PROYECCIÓN}

Dentro de los materiales comúnmente empleados como recubrimientos para aplicaciones biomédicas se encuentran los fosfatos de calcio, en particular la hidroxiapatita por su semejanza con la parte mineral del hueso; la Titania $\left(\mathrm{TiO}_{2}\right)$ por su biocompatibilidad; y en las últimas décadas los vidrios bioactivos, siendo el más popular para aplicaciones biomédicas el biovidrio 45S5 ${ }^{\mathrm{TM}}$ [4, 5, 25-27]. La hidroxiapatita se ha posicionado como uno de los materiales bioactivos más reportados, debido a su excelente comportamiento y estabilidad a largo plazo dentro del organismo; por otro lado, los vidrios bioactivos son materiales ampliamente atractivos para aplicaciones biomédicas, pues el biovidrio $45 \mathrm{~S} 5^{\mathrm{TM}}$ ha sido uno de los materiales que ha mostrado tener índices de comportamiento bioactivo más altos dentro de los biocerámicos. Además, esta familia de materiales ha sido el foco de investigaciones en años recientes, ya que, modificando sutilmente su composición es posible obtener cambios drásticos en sus propiedades. Actualmente en la literatura se encuentra poca información referente a la fabricación de recubrimientos cerámicos biocompatibles fabricados mediante proyección térmica sobre biopolímeros para aplicaciones ortopédicas, lo cual se revisará en la siguiente sección [25]. 


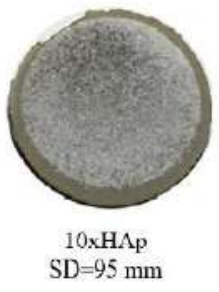

(a)

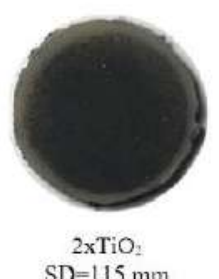

(b)

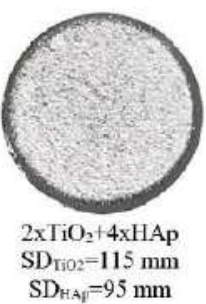

(c)
Figura 3. Fotografías de recubrimientos por APS obtenidos sobre PEEK. (a) 10 capas de HA.

(b) Recubrimiento de 2 capas de $\mathbf{T i O}_{2}$. (c) 2 capas de $\mathrm{TiO}_{2}$ y 4 de HA. En la imagen se puede apreciar la necesidad de un recubrimiento de anclaje para lograr la adhesión de la HA [28].

Tabla 2. Materiales usualmente empleados en recubrimientos ortopédicos

\begin{tabular}{|c|c|c|}
\hline Material & Características & Ref. \\
\hline $\begin{array}{c}\text { PEEK } \\
\text { (sustrato) }\end{array}$ & $\begin{array}{c}\text { Pobre osteointegración, } \\
\text { excelentes propiedades } \\
\text { mecánicas, estable física } \\
\text { y químicamente. }\end{array}$ & [29] \\
\hline $\begin{array}{c}\text { Titanio y } \\
\text { aleaciones } \\
\text { (sustrato) }\end{array}$ & $\begin{array}{c}\text { Excelentes propiedades } \\
\text { mecánicas (relación } \\
\text { fuerza-peso), resistencia } \\
\text { a la corrosión y } \\
\text { biocompatibilidad. }\end{array}$ & [30] \\
\hline $\begin{array}{c}\text { Buena osteointegración, } \\
\text { tasa lenta de } \\
\text { biodegradación, baja } \\
\text { tenacidad a la fractura y } \\
\text { estable a largo plazo. }\end{array}$ & {$[31]$} \\
\hline $\begin{array}{c}\text { Vidrio } \\
\text { bioactivo }\end{array}$ & $\begin{array}{c}\text { Frágil y débil, induce la } \\
\text { vascularización. }\end{array}$ & {$[32]$} \\
\hline & $\begin{array}{c}\text { Buena osteointegración, } \\
\text { buenas propiedades } \\
\text { Titania }\end{array}$ & [33] \\
\hline & $\begin{array}{c}\text { mecánicas, resistencia a } \\
\text { la corrosión. }\end{array}$ & \\
\hline
\end{tabular}

\section{MODIFICACIÓN SUPERFICIAL DE SUSTRATOS POLIMÉRICOS}

Existen varias técnicas de modificación superficial para proveer algunos polímeros con propiedades bioactivas, las cuales podemos ver de manera resumida en la Figura 5. Actualmente existen prótesis comerciales fabricadas de biopolímeros con recubrimientos cerámicos para cirugía intervertebral [34-36]. También se pueden encontrar biopolímeros reforzados con fibra de carbono (CFR-PEEK, siglas en inglés), los cuales, han sido empleados para fijación de fracturas $y$ prótesis femorales en articulaciones de cadera artificial[23].

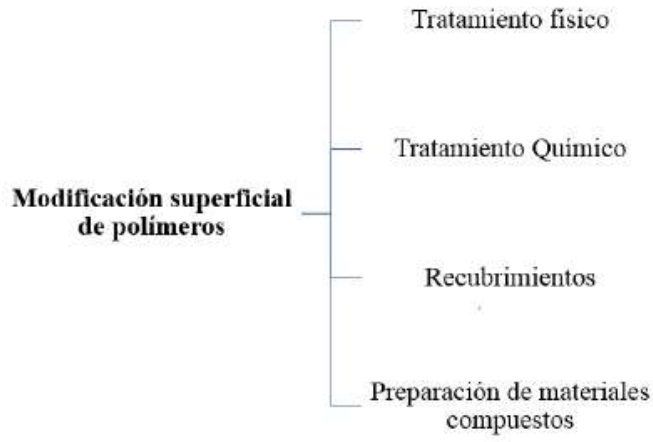

Figura 5. Métodos de modificación superficial de polímeros para mejorar sus propiedades bioactivas.

Al emplear tratamientos físicos se utilizan plasmas, los cuales pueden ser producidos en un sistema cerrado que contiene una mezcla de gases excitados por ondas electromagnéticas, es entonces, que las partículas generadas interactúan con la superficie del biopolímero y modifican su superficie química y físicamente, sin alterar otras propiedades (por ejemplo, propiedades mecánicas) [37, 38]. W. Ha et al. [39] modificaron la superficie del PEEK haciendo uso de dos técnicas de grabado; el químico y el plasma de oxígeno, pretendiendo obtener una superficie irregular con mayor rugosidad, para posteriormente fabricar un recubrimiento sumergiendo el PEEK (previamente modificado) en una solución supersaturada de fosfatos de calcio, para mejorar las propiedades de unión del PEEK con el hueso. Concluyeron que la modificación de superficie mediante plasma de oxígeno genera mejores condiciones que el grabado químico, para la formación de fosfatos de calcio. Awaja et al. [40, 41] 
trataron PEEK con plasma por radio frecuencia (RF) usando una mezcla de $\mathrm{CH}_{4} / \mathrm{O}_{2}$ encontraron que la fuerza de adhesión de la capa formada al ser sumergido en fluido biológico simulado (SBF, siglas en inglés) fue mucho mayor que en el polímero no modificado. Waser-Althaus et. al. [42], emplearon plasma con mezcla de $\mathrm{O}_{2} / \mathrm{Ar}$ para tratar la superficie, mostraron incrementos en adhesión y diferenciación osteogénica.

El tratamiento químico para la modificación superficial de polímeros se realiza usualmente mediante la hidroxilación, carboxilación, aminación o por hidrólisis del polímero, estas modificaciones químicas promueven la adhesión y proliferación de células óseas [23].

Los recubrimientos superficiales se fabrican mediante la deposición de distintos materiales (mencionados en la sección anterior de este documento) sobre la superficie del polímero, empleando distintas técnicas, por ejemplo, CS, APS, spin coating, aerosol deposition, magnetron sputtering, entre otros [43-45]. En la Tabla 3 podemos observar un resumen de trabajos realizados mediante la fabricación de recubrimientos por proyección térmica.

Tabla 3. Algunas modificaciones de superficie de polímeros mediante proyección térmica por plasma atmosférico (APS) y cold spray (CS).

\begin{tabular}{|c|c|c|c|}
\hline 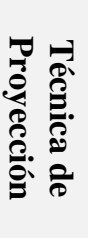 & 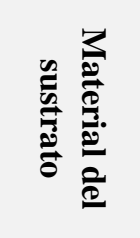 & 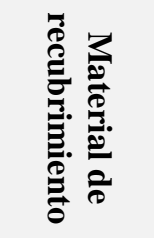 & 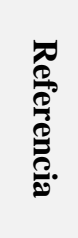 \\
\hline CS & $\begin{array}{l}\text { PEEK- } \\
\text { CFR }\end{array}$ & Aluminio & [46] \\
\hline $\mathrm{CS}$ & $\begin{array}{c}\text { Poliamid } \\
\text { a } 66\end{array}$ & Aluminio & [47] \\
\hline $\mathrm{CS}$ & $\begin{array}{c}\text { Poliureta } \\
\text { no }\end{array}$ & Cobre & [48] \\
\hline APS & $\begin{array}{c}\text { Epoxy- } \\
\text { CFR }\end{array}$ & Alúmina & [49] \\
\hline APS & $\begin{array}{c}\text { Poliamid } \\
\text { a-CFR }\end{array}$ & HA & [50] \\
\hline APS & PEEK & HA & [28] \\
\hline
\end{tabular}

\section{\begin{tabular}{|l|l|l|l|} 
APS & PEEK & HA & {$[26]$} \\
\hline
\end{tabular}}

La fabricación de recubrimientos mediante APS es mencionada en la literatura como la técnica más versátil dentro de las técnicas existentes dentro de la proyección térmica [6], por lo que, es la que más es empleada en distintas aplicaciones, y el caso de la fabricación de recubrimientos bioactivos sobre sustratos poliméricos no es la excepción. Cuando un material polimérico se pretende recubrir mediante proyección térmica, una de las características más importantes a evaluar es la adhesión del recubrimiento. Esto depende de varios factores, como la rugosidad del sustrato, la limpieza de éste, la diferencia entre coeficientes de expansión térmica de los materiales (sustrato y recubrimiento), los parámetros seleccionados en el proceso, entre otros. Que se logre realizar bien la configuración de los factores antes mencionados y se logre adherir el recubrimiento al sustrato depende de la fuerza de enlace, la cual es la cantidad de energía necesaria para desprender el recubrimiento del sustrato. Esta fuerza está conformada por las fuerzas de tensión internas $\left(\sigma_{\text {int }}\right)$ y la carga externa en el sistema ( $\left.\sigma_{\text {ext }}\right)$, la cual actúa principalmente en las uniones interfaciales entre el recubrimiento y el sustrato polimérico [10]. Las fuerzas de tensión interna se deben principalmente a las discrepancias existentes en el módulo elástico y coeficiente de expansión térmico entre el sustrato y el recubrimiento [51]. La fuerza interna que actúa en la interfaz (en el plano xy) está dada por:

$$
F_{\text {int }}=\int \sigma_{\text {int }}(z) w d z
$$

donde, w es el espesor de la muestra. Se puede decir que un recubrimiento proyectado por plasma muestra suficiente adhesión, si la fuerza de adhesión es mayor que la combinación de las fuerzas externas e 
internas actuando en la interfase como se muestra a continuación:

$$
F_{a d h}>\sigma_{\text {int }} d_{f i l m}+\frac{F_{\text {ext }} \sigma_{\text {ext }}}{w}
$$

donde, $F_{\text {adh }}$ es la fuerza de adhesión, $\mathrm{d}_{\text {film }}$ el espesor del recubrimiento, $F_{\text {ext }}$ la fuerza externa que actúa en la interfaz. Los valores típicos para una adhesión aceptable son de por lo menos 5-20 MPa (de acuerdo con la norma ASTM-633-01). El recubrimiento de sustratos poliméricos requiere adaptar los parámetros de procesamiento del plasma para lograr alcanzar la adhesión requerida sin dañar el sustrato. Barillas et al. [28], buscando conseguir una superficie bioactiva con dióxido de titanio como recubrimiento de anclaje (bond coat) e hidroxiapatita (HA) como recubrimiento superficial (top coat) sobre polietereterecetona (PEEK) mediante proyección térmica APS encontraron que la distancia de proyección y el enfriamiento del sustrato eran parámetros críticos para el proceso. Wu et al. [26], también fabricaron recubrimientos de HA sobre PEEK mediante proyección térmica APS. En su trabajo evaluaron dos polvos diferentes de HA y emplearon un sistema de monitoreo de la temperatura de superficie y velocidad de las partículas durante el proceso de rociado térmico (DPV 2000 Tecnar)encontraron que, en cuanto a la distribución y morfología de los polvos, de los dos tipos de HA, el que presentaba menor variación en la distribución de tamaño y morfología más esférica es el que es más favorable para la fabricación de los recubrimientos. Proponen la adición de un recubrimiento previo (bond coat) de titanio para mejorar la calidad del recubrimiento de HA. Obtuvieron recubrimientos con grosores de 30-40 $\mu \mathrm{m}$ con rugosidad de $4.9 \mu \mathrm{m}$. También haciendo uso del DPV 2000, evaluaron la influencia de la modificación de parámetros del proceso (distancia de proyección, corriente, flujo de gases y tasa de alimentación de polvos) en la velocidad de las partículas y eventualmente en la calidad del recubrimiento. Otra técnica de gran interés durante los últimos años ha sido la proyección en frio (CS), pues esta implica la aplicación de menor temperatura a las partículas, lo cual, puede ayudar a cuidar la integridad del sustrato. Mediante esta técnica se han fabricado también recubrimientos de HA sobre PEEK, haciendo pruebas in vitro e in vivo, concluyendo que el material puede tener posibles aplicaciones clínicas [27].

De acuerdo con los conceptos revisados hasta aquí, uno de los criterios más importantes para la obtención de un recubrimiento sobre la superficie de un biopolímero por cualquier técnica, es evitar la degradación del material. En particular, cuando nos enfrentamos con la proyección térmica, se deben tener en cuenta algunos de los principales parámetros en estos procesos como, por ejemplo, la velocidad y temperatura de las partículas, la rugosidad y temperatura del sustrato, el ángulo entre el chorro de proyección y el sustrato, la mezcla de gases utilizados, la potencia, la distancia de proyección, entre otros [9, 52, 53]. Es importante identificar los parámetros que pueden verse modificados en estos procesos para evitar afectaciones en las propiedades de materiales biopoliméricos a recubrir. Algunos grupos de investigadores se han centrado en identificar las diferencias en el procesamiento de recubrimientos bioactivos sobre sustratos poliméricos.

Otras alternativas con las que se ha experimentado para contrarrestar la propiedad bioinerte de los polímeros en aplicaciones biomédicas ha sido la fabricación de materiales compuestos, en su mayoría fabricando un compuesto, formado por el polímero base e hidroxiapatita. Mediante la implementación de HA se logra mejorar la bioactividad del material, sin embargo, las propiedades mecánicas se ven alteradas, esto es, la disminución de la ductilidad y la resistencia a la tracción y un 
aumento del valor del módulo elástico del compuesto $[54,55]$.

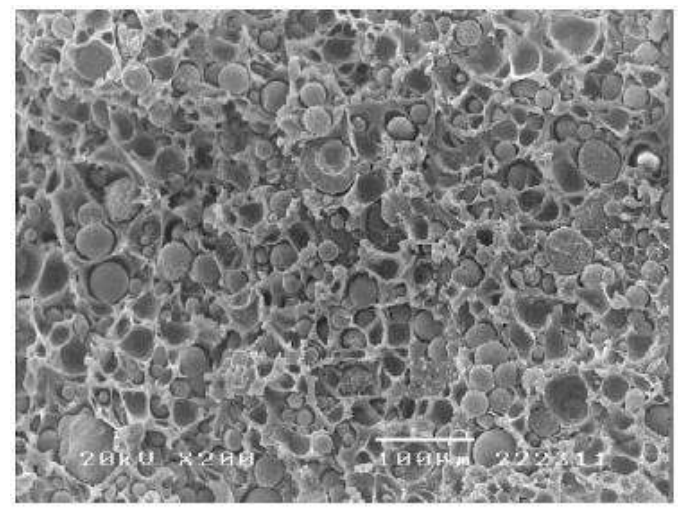

Figura 4. Compuesto de PEEK-HA (20\% en volumen) fabricado mediante moldeo por inyección [54]

\section{COMENTARIOS FINALES}

Los materiales poliméricos han sido ampliamente investigados y empleados en la industria biomédica. En particular, aquellos biopolímeros con propiedades mecánicas ideales para ser empleados en aplicaciones ortopédicas deben ser modificados superficialmente, para dotar a éstos de propiedades bioactivas. Existe gran variedad de técnicas de modificación superficial para este tipo de materiales, esto los convierte en materiales ampliamente atractivos para su uso en aplicaciones ortopédicas. En el caso particular de la sustitución de componentes metálicos han sido usados con éxito en ciertas zonas del cuerpo como en los discos vertebrales o implantes craneales; sin embargo, aún no han alcanzado completamente su potencial al ser utilizados como material núcleo para la fabricación de implantes bioactivos. A pesar de existir gran cantidad de trabajos referentes a la metalización y modificación de superficies poliméricas por diferentes técnicas, existe poca información en la literatura acerca de la modificación de superficies poliméricas con materiales bioactivos fabricados mediante proyección térmica. Los procesos de proyección térmica han sido empleados durante décadas para la fabricación de recubrimientos bioactivos sobre superficies metálicas y han mostrado cumplir mecánica y biológicamente en aplicaciones ortopédicas. Esta confianza adquirida por el buen desempeño de estos recubrimientos sobre implantes metálicos ha impulsado el desarrollo de recubrimientos por proyección térmica sobre materiales poliméricos. Esta funcionalización superficial parece ser reciente y se encuentran solo algunos desarrollos por proyección térmica por plasma y proyección fría. Estos trabajos muestran dificultades técnicas en cuanto a la obtención de los recubrimientos, principalmente, asociados a altas temperaturas y a altas velocidades de impacto de las partículas que resulta en bajas tasas de depósito y recubrimientos poco homogéneos. En este sentido, se intuye que en los trabajos de investigación actuales y futuros en este tema se busque controlar las condiciones de procesamiento como, por ejemplo, tasas de enfriamiento, velocidades de impacto o la evaluación de las distintas fuentes de energía térmica o cinética a fin de determinar las condiciones ideales para lograr recubrimientos biocerámicos homogéneos en sustratos poliméricos.

\section{AGRADECIMIENTOS}

Agradecimientos al Consejo Nacional de Ciencia y Tecnología de México CONACYT a través del proyecto 313990 "Simposio Nacional de Prótesis Ortopédicas: El estatus Actual". Al Dr. John Henao y a la dirección de Materiales Avanzados de CIATEQ A.C.

\section{REFERENCIAS}

X. FAN, J. CHEN, J. peng ZOU, Q. 
WAN, Z. cheng ZHOU, and J. ming RUAN, "Bone-like apatite formation on HA/316L stainless steel composite surface in simulated body fluid," Trans. Nonferrous Met. Soc. China (English Ed., vol. 19, no. 2, pp. 347352, Apr. 2009, doi: 10.1016/S10036326(08)60276-9.

[2] G. A. Clavijo-Mejía, J. A. HermannMuñoz, J. A. Rincón-López, H. Ageorges, and J. Muñoz-Saldaña, "Bovine-derived hydroxyapatite coatings deposited by highvelocity oxygen-fuel and atmospheric plasma spray processes: A comparative study," Surf. Coatings Technol., vol. 381, p. 125193, 2020, doi: 10.1016/j.surfcoat.2019.125193.

[3] M. Gardon, A. Latorre, M. Torrell, S. Dosta, J. Fernández, and J. M. Guilemany, "Cold gas spray titanium coatings onto a biocompatible polymer," Mater. Lett., vol. 106, pp. 97-99, 2013, doi: 10.1016/j.matlet.2013.04.115.

[4] A. S. Kang, G. Singh, and V. Chawla, "In-vitro performance of reinforced hydroxyapatite coatings deposited using vacuum plasma spray technique on Ti-6Al$4 V$," Mater. Today Proc., vol. 26, no. xxxx, pp. 671-676, 2019, doi: 10.1016/j.matpr.2019.12.363.

[5] S. M. Forghani, M. J. Ghazali, A. Muchtar, A. R. Daud, N. H. N. Yusoff, and C. H. Azhari, "Effects of plasma spray parameters on TiO2-coated mild steel using design of experiment (DoE) approach," Ceram. Int., vol. 39, no. 3, pp. 3121-3127, Apr. 2013, doi: 10.1016/j.ceramint.2012.09.092.

[6] P. L. Fauchais, J. V. R. Heberlein, and M. I. Boulos, Thermal Spray Fundamentals. 2014.

[7] L. Pawlowski, The Science and Engineering of Thermal Spray Coatings:
Second Edition. 2008.

[8] P. Fauchais and G. Montavon, "Thermal and cold spray: Recent developments," Key Eng. Mater., vol. 384, pp. 1-59, 2008, doi: 10.4028/www.scientific.net/kem.384.1.

[9] D. R. Tobergte and S. Curtis, Handbook of thermal spray technology, vol. 53, no. 9. 2013.

[10] D. Hegemann, Plasma Polymer Deposition and Coatings on Polymers, vol. 4, no. May 2014. 2014.

[11] B. D. Ratner, A. S. Hoffman, F. J. Schoen, and J. E. Lemons, An Introduction to Materials in Medicine. 2004.

[12] B. G. X. Zhang, D. E. Myers, G. G. Wallace, M. Brandt, and P. F. M. Choong, "Bioactive coatings for orthopaedic implants-recent trends in development of implant coatings" Int. J. Mol. Sci., vol. 15, no. 7, pp. 11878-11921, 2014, doi: 10.3390/ijms 150711878 .

[13] A. R. Cutler, S. Siddiqui, A. L. Mohan, V. H. Hillard, F. Cerabona, and K. Das, "Comparison of polyetheretherketone cages with femoral cortical bone allograft as a single-piece interbody spacer in transforaminal lumbar interbody fusion," $J$. Neurosurg. Spine, vol. 5, no. 6, pp. 534-539, 2006, doi: 10.3171/spi.2006.5.6.534.

[14] O. Noiset, Y. J. Schneider, and J. Marchand-Brynaert, "Fibronectin adsorption or/and covalent grafting on chemically modified PEEK film surfaces," J. Biomater. Sci. Polym. Ed., vol. 10, no. 6, pp. 657-677, 1999, doi: 10.1163/156856299X00865.

[15] L. Sedel, R. Nizard, and A. Meunier, Orthopedic biomaterials, vol. 179, no. 3 . 1995. 
[16] J. Y. Wong and J. D. Bronzino, Biomaterials. Boca Raton, FL: Taylor and Francis Group, 2007.

[17] L. Czuba, Application of Plastics in Medical Devices and Equipment. Elsevier Inc., 2014.

[18] A. Moridi, S. M. Hassani-Gangaraj, M. Guagliano, and M. Dao, "Cold spray coating: Review of material systems and future perspectives," Surf. Eng., vol. 30, no. 6, pp. 369-395, 2014, doi: 10.1179/1743294414Y.0000000270.

[19] MIT, "Material Properties Database MIT," PMMA. http://www.mit.edu/ 6.777/matprops/pmma. htm.

[20] J. Y. Rho, R. B. Ashman, and C. H. Turner, "Young's modulus of trabecular and cortical bone material: Ultrasonic and microtensile measurements," J. Biomech., vol. 26, no. 2, pp. 111-119, 1993, doi: 10.1016/0021-9290(93)90042-D.

[21] M. Arora, E. K. S. Chan, S. Gupta, and A. D. Diwan, "Polymethylmethacrylate bone cements and additives: A review of the literature," World J. Orthop., vol. 4, no. 2, pp. 67-74, 2013, doi: 10.5312/wjo.v4.i2.67.

[22] H. Wang et al., "Mechanical and biological characteristics of diamond-like carbon coated poly aryl-ether-ether-ketone," Biomaterials, vol. 31, no. 32, pp. 8181-8187, 2010 , doi: 10.1016/j.biomaterials.2010.07.054.

[23] R. Ma and T. Tang, "Current strategies to improve the bioactivity of PEEK," Int. J. Mol. Sci., vol. 15, no. 4, pp. 5426-5445, 2014, doi: 10.3390/ijms15045426.

[24] L. W. McKeen, Plastics Used in
Medical Devices. Elsevier Inc., 2014.

[25] J. Massera, S. Fagerlund, L. Hupa, and M. Hupa, "Crystallization mechanism of the bioactive glasses, 45 S5 and S53P4," J. Am. Ceram. Soc., vol. 95, no. 2, pp. 607-613, 2012, doi: $\quad 10.1111 / \mathrm{j} .1551-$ 2916.2011.05012.x.

[26] G. M. Wu, W. D. Hsiao, and S. F. Kung, "Investigation of hydroxyapatite coated polyether ether ketone composites by gas plasma sprays," Surf. Coatings Technol., vol. 203, no. 17-18, pp. 2755-2758, Jun. 2009, doi: 10.1016/j.surfcoat.2009.02.115.

[27] J. H. Lee et al., "In vitro and in vivo evaluation of the bioactivity of hydroxyapatite-coated polyetheretherketone biocomposites created by cold spray technology," Acta Biomater., vol. 9, no. 4, pp. 6177-6187, 2013, doi: 10.1016/j.actbio.2012.11.030.

[28] L. Barillas et al., "Bioactive Plasma Sprayed Coatings on Polymer Substrates Suitable for Orthopedic Applications: A Study With PEEK," IEEE Trans. Radiat. Plasma Med. Sci., vol. 2, no. 5, pp. 520-525, May 2018, doi: 10.1109/trpms.2018.2832450.

[29] N. T. Evans et al., "High-strength, surface-porous polyether-ether-ketone for load-bearing orthopedic implants," Acta Biomater., vol. 13, pp. 159-167, 2015, doi: 10.1016/j.actbio.2014.11.030.

[30] F. Visentin et al., " $\mathrm{TiO}_{2}-\mathrm{HA}$ bi-layer coatings for improving the bioactivity and service-life of Ti dental implants," Surf. Coatings Technol., vol. 378, p. 125049, 2019, doi: 10.1016/j.surfcoat.2019.125049.

[31] C. Zhou et al., "Mechanical and biological properties of the micro-/nanograin functionally graded hydroxyapatite bioceramics for bone tissue engineering," $J$. 
Mech. Behav. Biomed. Mater., vol. 48, pp. 111, 2015, doi: 10.1016/j.jmbbm.2015.04.002.

[32] E. Tamjid, R. Bagheri, M. Vossoughi, and A. Simchi, "Effect of particle size on the in vitro bioactivity, hydrophilicity and mechanical properties of bioactive glassreinforced polycaprolactone composites," Mater. Sci. Eng. C, vol. 31, no. 7, pp. 15261533, 2011, doi: 10.1016/j.msec.2011.06.013.

[33] M. B. Casu et al., "A multi-technique investigation of $\mathrm{TiO} 2$ films prepared by magnetron sputtering," Surf. Sci., vol. 602, no. 8, pp. 1599-1606, 2008, doi: 10.1016/j.susc.2008.02.030.

[34] T. Jiya, T. Smit, J. Deddens, and M. Mullender, "Posterior lumbar interbody fusion using nonresorbable poly-ether-etherketone versus resorbable poly-l-lactide-cod,l-lactide fusion devices: A prospective, randomized study to assess fusion and clinical outcome," Spine (Phila. Pa. 1976)., vol. 34, no. 3, pp. 233-237, 2009, doi: 10.1097/BRS.0b013e318194ed00.

[35] H. W. Park, J. K. Lee, S. J. Moon, S. K. Seo, J. H. Lee, and S. H. Kim, "The efficacy of the synthetic interbody cage and grafton for anterior cervical fusion," Spine (Phila. Pa. 1976)., vol. 34, no. 17, pp. 591595, 2009, doi: 10.1097/BRS.0b013e3181ab8b9a.

[36] J. M. Toth, M. Wang, B. T. Estes, J. L. Scifert, H. B. Seim, and A. S. Turner, "Polyetheretherketone as a biomaterial for spinal applications," Biomaterials, vol. 27, no. 3, pp. 324-334, 2006, doi: 10.1016/j.biomaterials.2005.07.011.

[37] D. Briem et al., "Response of primary fibroblasts and osteoblasts to plasma treated polyetheretherketone (PEEK) surfaces," J. Mater. Sci. Mater. Med., vol. 16, no. 7, pp.
671-677, 2005, doi: 10.1007/s10856-0052539-z.

[38] E. M. Liston, "Plasma treatment for improved bonding: A review," J. Adhes., vol. 30, no. 1-4, pp. 199-218, 1989, doi: $10.1080 / 00218468908048206$.

[39] S. W. Ha, R. Hauert, K. H. Ernst, and E. Wintermantel, "Surface analysis of chemically-etched and plasma-treated polyetheretherketone (PEEK) for biomedical applications," Surf. Coatings Technol., vol. 96, no. 2-3, pp. 293-299, 1997, doi: 10.1016/S0257-8972(97)00179-5.

[40] F. Awaja, S. Zhang, N. James, and D. R. McKenzie, "Enhanced Autohesive Bonding of Polyetheretherketone (PEEK) for biomedical applications using a methane/oxygen plasma treatment," Plasma Process. Polym., vol. 7, no. 12, pp. 10101021, 2010, doi: 10.1002/ppap.201000072.

[41] F. Awaja, D. V. Bax, S. Zhang, N. James, and D. R. McKenzie, "Cell adhesion to PEEK treated by plasma immersion ion implantation and deposition for active medical implants," Plasma Process. Polym., vol. 9, no. 4, pp. 355-362, 2012, doi: 10.1002/ppap.201100034.

[42] J. Waser-Althaus et al., "Differentiation of human mesenchymal stem cells on plasma-treated polyetheretherketone," J. Mater. Sci. Mater. Med., vol. 25, no. 2, pp. 515-525, 2014, doi: 10.1007/s10856-013-5072-5.

[43] S. Barkarmo et al., "Nanohydroxyapatite-coated PEEK implants: A pilot study in rabbit bone," J. Biomed. Mater. Res. - Part A, vol. 101 A, no. 2, pp. 465-471, 2013, doi: 10.1002/jbm.a.34358.

[44] B. D. Hahn et al., "Osteoconductive hydroxyapatite coated PEEK for spinal 
fusion surgery," Appl. Surf. Sci., vol. 283, pp. 6-11, 2013, doi: 10.1016/j.apsusc.2013.05.073.

[45] A. Rabiei and S. Sandukas, "Processing and evaluation of bioactive coatings on polymeric implants," J. Biomed. Mater. Res. - Part A, vol. 101 A, no. 9, pp. 2621-2629, 2013, doi: 10.1002/jbm.a.34557.

[46] A. Sturgeon, B. Dunn, S. Celotto, and B. O'Neill, "Cold sprayed coatings for polymer composite substrates," Eur. Sp. Agency, (Special Publ. ESA SP, vol. 2006, no. 616 , pp. 19-23, 2006.

[47] D. Giraud, F. Borit, V. Guipont, M. Jeandin, and J. M. Malhaire, "Metallization of a polymer using cold spray: Application to aluminum coating of polyamide 66," Proc. Int. Therm. Spray Conf., no. February, pp. 265-270, 2012.

[48] P. Taylor, M. J. Vucko, P. C. King, A. J. Poole, M. Z. Jahedi, and R. De Nys, "Biofouling: The Journal of Bioadhesion and Biofilm Polyurethane seismic streamer skins : an application of cold spray metal embedment," no. January 2013, pp. 37-41, 2013.

[49] G. Sun, X. He, J. Jiang, Y. Sun, and Y. Zhong, "A study on the deposition of $\mathrm{Al}_{2} \mathrm{O}_{3}$ coatings on polymer substrates by a plasma spray/micro-arc oxidation two-step method," J. Therm. Spray Technol., vol. 22, no. 1, pp. 27-35, 2013, doi: 10.1007/s11666-012-98658 .

[50] C. Auclair-Daigle, M. N. Bureau, J. G. Legoux, and L. Yahia, "Bioactive hydroxyapatite coatings on polymer composites for orthopedic implants," J. Biomed. Mater. Res. - Part A, vol. 73, no. 4, pp. 398-408, 2005, doi: 10.1002/jbm.a.30284.

[51] S. Tamulevičius, "Stress and strain in the vacuum deposited thin films," Vacuum, vol. 51, no. 2, pp. 127-139, 1998, doi: 10.1016/S0042-207X(98)00145-6.

[52] P. Fauchais, M. Fukumoto, A. Vardelle, and M. Vardelle, "Knowledge concerning splat formation: An invited review," J. Therm. Spray Technol., vol. 13, no. 3, pp. 337-360, 2004, doi: 10.1361/10599630419670.

[53] R. Gonzalez, H. Ashrafizadeh, A. Lopera, P. Mertiny, and A. McDonald, " $A$ Review of Thermal Spray Metallization of Polymer-Based Structures," J. Therm. Spray Technol., vol. 25, no. 5, pp. 897-919, 2016, doi: 10.1007/s11666-016-0415-7.

[54] M. S. Abu Bakar et al., "Tensile properties, tension-tension fatigue and biological response of polyetheretherketonehydroxyapatite composites for load-bearing orthopedic implants," Biomaterials, vol. 24, no. 13, pp. 2245-2250, 2003, doi: $10.1016 / \mathrm{S} 0142-9612(03) 00028-0$.

[55] K. L. Wong et al., "Mechanical properties and in vitro response of strontiumcontaining

hydroxyapatite/polyetheretherketone composites," Biomaterials, vol. 30, no. 2324, pp. 3810-3817, 2009, doi: 10.1016/j.biomaterials.2009.04.016. 